\title{
International Prevalence of the Use of Peripheral Intravenous Catheters
}

\author{
Evan Alexandrou, RN, BHealth, ICU Cert, MPH, PhDD1,2,3*, Gillian Ray-Barruel, RN, BSN, BA, ICU Cert 3 , \\ Peter J. Carr, RN, Dip HE Nurs, H Dip A\&E Spec Nurs, BSc, MMedSc ${ }^{3,4,5}$, Steven Frost, RN, ICU Cert, MPH, PhD 1,2, \\ Sheila Inwood, RN, CNS ${ }^{6,7}$, Niall Higgins, RN, GDipeH, $\mathrm{PhD}^{3}$, Frances Lin, RN, $\mathrm{PhD}^{3}$, Laura Alberto, RN ${ }^{8}$, \\ Leonard Mermel, DO, ScM, AM, FACP, FIDSA, FSHEA ${ }^{9}$, Claire M. Rickard, RN, GradDip N(CritCare), PhD, FACN 3,4
}

\begin{abstract}
'School of Nursing and Midwifery, University of Western Sydney, Penrith, New South Wales, Australia; 'Intensive Care, Liverpool Hospital, Sydney, New South Wales, Australia; ${ }^{3}$ Alliance for Vascular Access Teaching and Research Group, Menzies Health Institute Queensland, Centre for Health Practice Innovation, Griffith University, Nathan, Queensland, Australia; ${ }^{4}$ Australian National Health and Medical Research Council, Centre of Research Excellence in Nursing Interventions, Gold Coast Campus, Queensland, Australia; ${ }^{5}$ Emergency Medicine, The University of Western Australia, Perth, Western Australia, Australia; ${ }^{6}$ CareFusion, Rolle, Switzerland; ${ }^{7}$ Royal Berkshire Hospital, Reading, United Kingdom; ${ }^{8}$ Sanatorio de Alta Complejidad Sagrado Corazón, Buenos Aires, Argentina; ${ }^{9}$ Rhode Island Hospital and Warren Alpert Medical School of Brown University, Providence, Rhode Island, USA.
\end{abstract}

Over a billion peripheral intravenous catheters (PIVCs) are inserted each year in hospitalized patients worldwide. However, international data on prevalence and management of these devices are lacking. The study assessed the prevalence of PIVCs and their management practices across different regions of the world. This global audit involved 14 hospitals across 13 countries, with 479 patients screened for the presence of a PIVC. We found $59 \%$ of patients had at least 1 PIVC in place, and $16 \%$ had other types of vascular devices. We also found that overall, $25 \%$ of patients had no vascular device in place. The majority of PIVCs were inserted by nursing staff or a specialist team. The prevalence of idle PIVCs in place with no fluid or medication orders was $16 \%$, and $12 \%$ of PIVCs had at least 1 symptom of phlebitis. Journal of Hospital Medicine 2015;10:530-533. (C) 2015 Society of Hospital Medicine
Peripheral intravenous catheters (PIVCs) are ubiquitous devices that can have serious complications including bloodstream infections. ${ }^{1}$ The annual use of PIVCs in North America has been reported to be in excess of 330 million. The estimated number of PIVCs used across greater Europe or other regions of the world is largely unknown, although estimates from global device sales have been reported to be approximately 1.2 billion. ${ }^{1,2}$

Robust data on the prevalence of PIVCs and their associated management and infection prevention practices remain poor in Western countries; even more concerning is that PIVC data in developing nations remain relatively unknown. ${ }^{3}$ Healthcare-associated infection rates are significantly higher in developing nations, where the lack of resources and staff training can contribute to poor PIVC insertion and management. ${ }^{4,5}$

There are currently scant data on PIVC management practices across different regions of the world. Localized complication rates such as phlebitis and infiltration are an under-reported problem, yet are known to be a contributing factor for PIVC failure that leads to premature cessation of intravenous (IV)

\footnotetext{
*Address for correspondence and reprint requests: Evan Alexandrou, University of Western Sydney, Building EB, Ground Level Room 44, Parramatta South Campus, Locked Bag 1797, Penrith South DC 1797, New South Wales 2751, Australia; Telephone: +612-9685-9506; Fax: +6129685-9023; E-mail: e.alexandrou@uws.edu.au

Additional Supporting Information may be found in the online version of this article.

Received: December 16, 2014; Revised: April 19, 2015; Accepted: April 26, 2015

2015 Society of Hospital Medicine DOI 10.1002/jhm.2389

Published online in Wiley Online Library (Wileyonlinelibrary.com).
}

therapy, device removal, and the requirement for resiting of a new PIVC. Such failure can lead to delays in IV therapy, increased length of hospital stay, and cost. ${ }^{6}$ Importantly, it can also lead to patient-reported anxiety and pain. This lack of information has made it difficult to identify contributing factors for PIVC failure that may include inserter characteristics, patient-related factors, and anatomical placement as well as healthcare facility adherence to international best practice and infection prevention guidelines. ${ }^{6,7}$

The aim of this study was to undertake a multicenter, international study to assess the prevalence of PIVCs across different countries, to review population and PIVC characteristics from different regions of the world, and ascertain whether a larger study would provide beneficial data. The data of interest for this study included: (1) prevalence of PIVC use, (2) patient and PIVC characteristics, (3) prevalence of localized symptoms such as phlebitis, and (4) PIVC securement and dressing practices.

\section{MATERIALS AND METHODS Study Design and Participants}

Participating hospitals were sourced through the authors' international networks and specialist organizations in vascular access (such as the Association for Vascular Access in the United States and the World Congress in Vascular Access in Europe). A convenience sampling method was used for this point prevalence study. Participating sites were instructed to choose inpatient wards with medical or surgical patients and were asked to collect data on as many patients as possible with a PIVC in place on a given 


\begin{tabular}{|c|c|c|c|c|}
\hline Region/Country & $\begin{array}{l}\text { PIVC, } \\
n(\%)\end{array}$ & $\begin{array}{c}\text { Other } \\
\text { VAD, } n(\%)\end{array}$ & $\begin{array}{c}\text { No } \\
\text { IV, } n(\%)\end{array}$ & $\begin{array}{c}\text { Total } \\
\text { Patients, } \mathrm{n}\end{array}$ \\
\hline \multicolumn{5}{|l|}{ North America } \\
\hline Canada & $10(48)$ & $11(52)$ & 0 & 21 \\
\hline United States of America & $16(64)$ & $9(36)$ & 0 & 25 \\
\hline \multicolumn{5}{|l|}{ Latin America } \\
\hline Argentina & $50(79)$ & $3(5)$ & $10(16)$ & 63 \\
\hline \multicolumn{5}{|l|}{ Western Europe } \\
\hline England & $23(100)$ & 0 & 0 & 23 \\
\hline Greece & $5(71)$ & $2(29)$ & 0 & 7 \\
\hline |taly & $12(34)$ & $9(26)$ & $14(40)$ & 35 \\
\hline Malta & $18(78)$ & 0 & $5(22)$ & 23 \\
\hline Scotland & $12(100)$ & 0 & 0 & 12 \\
\hline Spain & $59(83)$ & $3(4)$ & $9(13)$ & 71 \\
\hline \multicolumn{5}{|l|}{ Asia } \\
\hline China & $23(24)$ & $24(26)$ & $46(50)$ & 93 \\
\hline India & $16(73)$ & $2(9)$ & $4(18)$ & 22 \\
\hline \multicolumn{5}{|l|}{ Oceania } \\
\hline Australia & $18(37)$ & $13(26)$ & $18(37)$ & 49 \\
\hline New Zealand & $19(54)$ & 0 & $16(46)$ & 35 \\
\hline
\end{tabular}

NOTE: Other VAD indicates any intravenous device that is not a PIVC. Abbreviations: IV, intravenous; PIVC, peripheral intravenous catheter; VAD, vascular access device.

day. This method of patient recruitment was used due to the nature of the collaboration with participating sites; workload constraints dictated final sample numbers, as no funding was available. Sampling of general medical or surgical patients was expected to yield the greatest number of PIVCs compared to higher acuity areas.

The study was approved by the Human Research Ethics Committee of Griffith University (Queensland, Australia), with each participating organization required to comply with local ethical and regulatory requirements prior to participation. For the purpose of the study, only adult patients were screened, and all were required to give verbal or written informed consent prior to assessment of the PIVC.

A site questionnaire identified organizational characteristics regarding resource allocation and clinician training for insertion and management of PIVCs. The patient case report form (CRF) elicited information on patient demographics, characteristics of the PIVC, site assessment, and dressing and securement assessment. The CRF provided standardized assessment criteria. The Strengthening the Reporting of Observational Studies in Epidemiology guidelines for cross-sectional studies were followed, and results are presented following these recommendations.

\section{Statistical Analysis}

Statistical software (SAS version 9.1; SAS Institute, Inc., Cary, NC) was used with results stratified into individual countries and regions. Proportions were used (with total number of PIVCs as the denominator) to present the data on PIVC characteristics. Data describing the prevalence of PIVC by country used individual country totals for derivation of a denominator.

\section{RESULTS}

\section{Prevalence of PIVC Use}

Fourteen sites in 13 countries contributed to this study (including 2 sites in the United States). The regions of Oceania, North and South America, Europe, and Asia were all represented. A total of 479 patients across all sites were screened for the presence of a PIVC. On the day of the study, the PIVC prevalence was $59 \%$ $(\mathrm{n}=281)$, with a range of $24 \%$ to $100 \%$; only 1 patient across the entire cohort had more than 1 PIVC in place on the day of the study. The prevalence of patients with a vascular access device (VAD) other than a PIVC (eg, centrally or peripherally inserted central venous catheters) was $16 \%(\mathrm{n}=76)$, and a quarter $(\mathrm{n}=122,25 \%)$ of the patients screened had no VAD in place (Table 1 ).

The study sites in Spain and Argentina were among the countries that screened the largest number of patients and had a similar prevalence of PIVC use $(83 \%$ and $79 \%$, respectively, Table 1$)$. The study site in China, which screened the highest number of patients overall $(\mathrm{n}=93)$, had the lowest PIVC prevalence at only $24 \%$; this site also had the highest proportion of patients with no device at all $(50 \%)$.

\section{PIVC Characteristics}

Overall, PIVC gauge preference was between 18 gauge and 22 gauge; this comprised $95 \%$ of all PIVCs in place across the regions. The forearm was the preferential choice for the regions of North America and Asia, with approximately half of PIVCs placed in this area. Notably, most PIVCs were inserted by nurses or specialty vascular access teams (Table 2), with medical practitioner insertions reported in only 2 regions (Western Europe and Oceania). Overall, most PIVCs were inserted in the general wards (91\%). No PIVCs were found to have been inserted in the emergency room on the day of the study, although they could be represented in the unknown category.

There were disparate results across the regions for whether patients had a documented IV fluid order or IV medication order. The Asian region had the highest proportion of documented IV fluid and medication orders ( $85 \%$ and $95 \%$, respectively) for patients with a PIVC. The lowest proportions of documented IV fluid and medication orders were from Oceania $(38 \%$ and $43 \%$, respectively). This region also had the highest number of PIVCs with neither IV nor medication order $(43 \%)$. The overall study incidence of redundant PIVCs with no IV orders was $16 \%$.

Most PIVC sites assessed had no symptoms of phlebitis; although every region had some patients with at least 1 sign (range: 3\%-12\%). PIVC dressings were primarily clean and intact $(\mathrm{n}=226,80 \%)$; however, the Oceania region had the highest proportion of dressings that were loose or lifting (24\%). Dressing selection was homogenous in North America, Latin America, and Asia, where study sites exclusively used 
TABLE 2. Characteristics of Patients and PIVCs

\begin{tabular}{|c|c|c|c|c|c|c|}
\hline \multirow[b]{2}{*}{ Population Group* } & \multicolumn{6}{|c|}{ Region } \\
\hline & North America & Latin America & Western Europe & Asia & Oceania & Total \\
\hline Total PIVCS, n (\%) & $26(9)$ & $50(18)$ & $129(46)$ & $39(14)$ & $37(13)$ & $281(100)$ \\
\hline Age, mean (SD), y & $58(16)$ & $51(17)$ & $66(19)$ & $51(19)$ & $68(17)$ & $59(18)$ \\
\hline Total men, $n(\%)$ & $11(42)$ & $26(52)$ & $72(56)$ & $19(49)$ & $25(68)$ & $154(55)$ \\
\hline \multicolumn{7}{|l|}{ Hospital category, n (\%) } \\
\hline Medical & $23(89)$ & $19(38)$ & $96(74)$ & $28(72)$ & $13(35)$ & $179(63)$ \\
\hline Surgical & 0 & $23(46)$ & $30(23)$ & $3(8)$ & $16(43)$ & $72(26)$ \\
\hline Oncology & 0 & 0 & $3(2)$ & $8(20)$ & $8(22)$ & $19(7)$ \\
\hline Intensive/coronary care & $3(11)$ & $8(16)$ & 0 & 0 & 0 & $11(4)$ \\
\hline \multicolumn{7}{|l|}{ PIVC inserted by, n (\%) } \\
\hline Specialist team & $14(54)$ & $8(16)$ & $19(15)$ & 0 & $27(72)$ & $68(24)$ \\
\hline Nurse & $12(47)$ & $42(84)$ & $88(68)$ & $39(100.0)$ & $2(5.0)$ & $183(65)$ \\
\hline Doctor & 0 & 0 & $22(17)$ & 0 & $7(19)$ & $29(10)$ \\
\hline Technician & 0 & 0 & 0 & 0 & $1(3)$ & $1(1)$ \\
\hline \multicolumn{7}{|l|}{ Where PIVC was inserted, $n(\%)$} \\
\hline Ward & $22(85)$ & $42(84)$ & $124(96)$ & $39(100.0)$ & $28(76)$ & $255(91)$ \\
\hline Intensive/coronary care & $3(12)$ & $8(16)$ & 0 & 0 & 0 & $11(4)$ \\
\hline Unknown & $1(4)$ & 0 & $5(4)$ & 0 & $9(24)$ & $15(5)$ \\
\hline \multicolumn{7}{|l|}{ Current IV fluid orders, n (\%) } \\
\hline Yes & $10(38)$ & $27(54)$ & $62(48)$ & $33(85)$ & $14(38)$ & $146(52)$ \\
\hline \multicolumn{7}{|l|}{ Current IV meds orders, n (\%) } \\
\hline Yes & $22(85)$ & $40(80)$ & $93(72)$ & $37(95)$ & $16(43)$ & $208(74)$ \\
\hline \multicolumn{7}{|l|}{ No IV or meds order, n (\%) } \\
\hline Yes & $3(12)$ & $6(12)$ & $20(16)$ & $1(3)$ & $16(43)$ & $46(16)$ \\
\hline \multicolumn{7}{|l|}{ Dressing quality, $n(\%)$} \\
\hline Clean and intact & $25(96)$ & $43(86)$ & $98(76)$ & $35(90)$ & $25(68)$ & $226(80)$ \\
\hline Moist or soiled & 0 & $2(4)$ & $17(13)$ & $1(2)$ & $3(8)$ & $23(8)$ \\
\hline Loose or lifting & $1(4)$ & $5(10)$ & $14(11)$ & $3(8)$ & $9(24)$ & $32(12)$ \\
\hline \multicolumn{7}{|l|}{ Symptoms of phlebitis, $n(\%)$} \\
\hline None & $25(96)$ & $44(88)$ & $114(88)$ & $32(82)$ & $36(97)$ & $251(89)$ \\
\hline Pain or tenderness & 0 & $3(6)$ & $5(4)$ & 0 & 0 & $8(3)$ \\
\hline Redness & 0 & 0 & $7(5)$ & $2(5)$ & 0 & $9(3)$ \\
\hline Swelling & $1(4)$ & $3(6)$ & $2(2)$ & $1(3)$ & 0 & $7(3)$ \\
\hline Other & 0 & 0 & $1(1)$ & $4(10)$ & $1(3)$ & $6(2)$ \\
\hline
\end{tabular}

NOTE: Abbreviations: IV, intravenous; PIVC, peripheral intravenous catheter; SD, standard deviation. ${ }^{*}$ Population group: North America = Canada and United States of America; Latin America = Argentina; Western Europe = England, Greece, Italy, Malta, Scotland, and Spain; Asia = China and India; Oceania = Australia and New Zealand.

borderless transparent polyurethane dressings. A small proportion $(9 \%)$ of patients in Western Europe had gauze and tape dressings.

Five of the 14 sites (36\%) had a dedicated IV team, and most hospitals had dedicated PIVC insertion training for nursing staff $(\mathrm{n}=10,71 \%)$. In contrast, only $43 \% \quad(n=6)$ of sites provided PIVC insertion training for medical staff. Some facilities also used specially trained technicians to undertake cannulation $(\mathrm{n}=6,43 \%)$. Most sites had policies for care and maintenance of PIVCs $(\mathrm{n}=12,86 \%)$ and predominantly prescribed routine replacement of PIVCs every 72 to 96 hours $(\mathrm{n}=11,83 \%)$. No sites exclusively prescribed leaving PIVCs in place until clinically indicated for removal, although some provided this as an option for certain patients.

\section{DISCUSSION}

This study has shown variation in the prevalence, characteristics, and management practices of PIVCs across sites from different regions of the world. Estimates for global PIVC prevalence in hospitalized patients vary widely from $30 \%$ to $80 \% .^{8-10}$ The overall prevalence of PIVCs in this pilot study at $59 \%$ lay in the midrange of those reported in recent literature, ${ }^{11}$ yet we found disproportionate PIVC prevalence between sites and regions. This heterogeneity could be explained by a number of factors including cohort acuity, clinician preference, and hospital guidelines. The generalizability of results from participating hospitals to their country is limited, because in most countries only a single institution participated.

Insertion of PIVCs was mainly by nurses, except in the Oceania region, where specialist teams and medical staff were the primary inserters. Of concern was the disparity in training provided by sites, with medical staff being less likely to receive instruction in how to prevent infection during this important procedure. A larger study would be needed to understand the effect on patient and infection outcomes of different inserter models and training provided.

A small proportion of patients from a site in Western Europe were observed to have gauze and tape as the PIVC dressing. The preference for gauze and tape 
is not common in developed nations, although recommended in clinical practice guidelines as an acceptable option. ${ }^{12}$ There is currently no strong evidence to suggest that any 1 dressing or securement device to secure PIVCs is more effective than any other. ${ }^{13}$ Nearly a quarter of PIVCs were loose or lifting from the Oceania region, this is of concern as interrupted dressings have been shown to increase the risk of catheter failure and catheter-related bloodstream infection. ${ }^{14}$

We found that $17 \%$ of PIVCs overall had no IV order for fluids or medication. This proportion of "redundant" catheters increases the burden of preventable intravascular infection. ${ }^{15}$ The prevalence of unnecessary PIVCs was lowest in Asia and greatest in the Oceania region, where $43 \%$ had no documented IV orders.

We reported PIVC prevalence from only a small number of nonrepresentative international sites for the purpose of considering a larger prevalence study. Observed differences in PIVC care and management cannot be generalized to entire regions. We asked sites to focus on medical-surgical wards, and as such some PIVCs in higher acuity areas were likely not included. A larger study will help to assess PIVC outcomes and contributing factors for any differences, and improve external validity.

Operational challenges also may have affected sample selection and size. This was an unfunded study undertaken by hospital investigators, with competing workload demands. Poor or slow internet connection at the bedside was reported by every participating site, and may have contributed to the small numbers of patients screened at some sites.

\section{CONCLUSION}

More than half of hospitalized patients screened internationally had a PIVC, and 1 in 4 patients had no VAD, with wide variability from country to country both in prevalence and practice. The data gained have provided valuable initial insights into the global variation in PIVC use and care, and confirm that a larger international study with multiple sites is warranted. In particular, it remains important to understand variations in PIVC use and whether country or regional trends increase the risk of infection.

\section{Acknowledgements}

The authors thank the following collaborators for assisting in the collection of the data for this pilot study: Argentina: Laura Alberto, Fabio Castel, Estela Farias, and Carlos Díaz; Australia: Nicholas Mifflin and Timothy Spencer; Canada: Jocelyn Hill; China: Lili Jin; Greece: Evangelos Konstantinou and Theodoros Katsoulas; India: Gracy Joseph and Sojan Ipe; Italy: Giancarlo Scoppettuolo and Laura Dolcetti; Malta: Michael Borg and Elmira Tartari; New Zealand: Ruth Barratt; Scotland: Linda Kelly and Audrey Green; Spain: Sonia Casanova, José Luis Micó, and Vicenta Solaz; England: Sheila Inwood; United States: Julie Jefferson and Janette Whitley.
Disclosures: All authors have made substantial contributions to the study conception and design, acquisition of the data, and analysis and interpretation of the data. Each author has contributed to drafting and editing the manuscript and approved the final version for publishing as per the International Committee of Medical Journal Editors convention. The authors wish to declare they have received unrestricted investigatorinitiated research grants from Becton Dickinson (BD) and 3M. The investigators also received professional translation services for most languages that were funded by B. Braun. All funds have been made payable to Griffith University and not to researchers themselves. These funders played no role in the conception, design, execution, analysis or reporting of the study. BD, 3M, CareFusion, Smiths Medical, B Braun, Vygon, and Teleflex assisted in disseminating study information and assisting with translation of data forms where necessary. No commercial entity had any involvement in the design, execution and analysis, or reporting of the study. Evan Alexandrou has provided education services for CareFusion, Teleflex, Cook Medical, and 3M. Peter Carr is undertaking a $\mathrm{PhD}$, which is partly funded by $\mathrm{BD}$. He has received payment for educational lectures from CareFusion. Claire Rickard's department (Griffith University) has received investigator-initiated, unrestricted research/educational grants from suppliers of vascular access device products including: 3M, BD, CareFusion, and Centurion. Claire Rickard has undertaken contract research or educational lectures for Bard, BBraun, $\mathrm{BD}$, CareFusion, and Teleflex. Sheila Inwood is an employee of CareFusion. Leonard Mermel has received research funding from Theravance, Astellas Pharma, Marvao Medical, and CareFusion, and he has been a consultant for 3M, CareFusion, Catheter Connections, Fresenius Medical, Marvao Medical, Bard Access, and ICU Medical.

\section{References}

1. Zingg W, Pittet D. Peripheral venous catheters: an under-evaluated problem. Int J Antimicrob Agents. 2009;34:S38-S42.

2. PR Newswire. Global peripheral I.V. catheter market 2014-2018. Available at: http://www.prnewswire.com/news-releases/globalperipheral-iv-catheter-market-2014-2018-257019061.html. Accessed April 28, 2015.

3. Allegranzi B, Nejad SB, Combescure C, et al. Burden of endemic health-care-associated infection in developing countries: systematic review and meta-analysis. Lancet. 2011;377(9761):228-241.

4. Rosenthal VD, Guzman S, Migone O, Crnich CJ. The attributable cost, length of hospital stay, and mortality of central line-associated bloodstream infection in intensive care departments in Argentina: a prospective, matched analysis. Am J Infect Control. 2003;31(8):475480.

5. Nejad SB, Allegranzi B, Syed SB, Ellis B, Pittet D. Health-care-associated infection in Africa: a systematic review. Bull World Health Organ. 2011;89(10):757-765.

6. Wallis MC, McGrail MR, Webster J, Gowardman JR, Playford G, Rickard CM. Risk factors for PIV catheter failure: a multivariate analysis from a randomized control trial. InfectControl Hosp Epidemiol. 2014;35(1):63-68.

7. Kagel EM, Rayan GM. Intravenous catheter complications in the hand and forearm. J Trauma. 2004;56(1):123-127.

8. Ritchie S, Jowitt D, Roberts S. The Auckland City Hospital Device Point Prevalence Survey 2005: utilisation and infectious complications of intravascular and urinary devices. NZ Med J. 2007;120(1260): U2683.

9. Malach T, Jerassy Z, Rudensky B, et al. Prospective surveillance of phlebitis associated with peripheral intravenous catheters. Am J Infect Control. 2006;34(5):308-312.

10. Pujol M, Hornero A, Saballs M, et al. Clinical epidemiology and outcomes of peripheral venous catheter-related bloodstream infections at a university-affiliated hospital. J Hosp Infect. 2007;67(1):22-29.

11. Webster J, Gillies D, O’Riordan E, Sherriff KL, Rickard CM. Gauze and tape and transparent polyurethane dressings for central venous catheters. Cochrane Database Syst Rev. 2011;(11):CD003827.

12. Gillies D, O'Riordan L, Carr D, Frost J, Gunning R, O'Brien I. Gauze and tape and transparent polyurethane dressings for central venous catheters. Cochrane Database Syst Rev. 2003;(4):CD003827.

13. Gillies D, O'Riordan E, Carr D, O’Brien I, Frost J, Gunning R. Central venous catheter dressings: a systematic review. I Adv Nurs. 2003; 44(6):623-632.

14. Timsit J-F, Bouadma L, Ruckly S, et al. Dressing disruption is a major risk factor for catheter-related infections. Crit Care Med. 2012;40(6): 1707-1714.

15. Lederle FA, Parenti CM, Berskow LC, Ellingson KJ. The idle intravenous catheter. Ann Intern Med. 1992;116(9):737-738. 